\title{
Inovação tecnológica, relação universidade-empresa e modelo teórico da Hélice Tripla
}

\author{
Gustavo Alberto Silva Coutinho1; André Vasconcelos da Silva²
}

\section{Resumo}

O presente artigo de revisão de literatura teve como objetivo realizar um recorte da produção científica a respeito da inovação tecnológica como vantagem competitiva, papel das universidades nesse contexto e a relação destas com as empresas para transferência de tecnologia, no contexto do modelo teórico da Hélice Tripla, proposto por Etzkowitz e Leydesdorff (2000). Para tanto, foram utilizados como referências artigos científicos que tratam dos temas apresentados e recentemente publicados. Como resultado, tem-se que no Brasil as empresas apresentam características próprias que dificultam o investimento em pesquisas, o que acaba por concentrar nas pesquisas realizadas pelas universidades a maior parte da produção de inovações tecnológicas. O modelo teórico da Hélice Tripla de relacionamento entre universidades, empresas e Estado, tem como finalidade criar ambiente favorável para aproximação entre universidades e empresas e facilitar a transferência de tecnologia da academia para o mercado.

Palavras-chave: inovação; relação universidade-empresa; Hélice Tripla

\section{INTRODUÇÃO}

O presente trabalho constitui uma revisão teórica acerca da interação universidade-empresa para produção de inovações tecnológicas. Para tanto, foi feito um levantamento de artigos publicados que tratam de tal tema, além de uma análise de dados estatísticos divulgados pelo CNPq, pelo SEBRAE e pelo IBGE.

Também se procedeu uma análise do artigo original de Etzkowitz e Leydesdorff (2000), em que os autores propõe o chamado modelo teórico da Hélice Tripla para analisar a interação entre universidade-empresa e o papel do Estado na mesma interação.

A partir de tais análises de informações estatísticas e do modelo teórico da Hélice Tripla, apresenta-se um recorte teórico que revela as características das empresas brasileiras e o papel das universidades na produção de inovações tecnológicas no país.

\footnotetext{
${ }^{1}$ Mestrando em Gestão Organizacional da Universidade Federal de Goiás; professor de Direito Empresarial no Centro de Ensino Superior de Catalão - CESUC; pesquisa financiada pela FAPEG.

2 Doutor em Ciências do Comportamento; Coordenador do Laboratório de Análise Organizacional e Consumo da Universidade Federal de Goiás.
} 


\section{DESENVOLVIMENTO}

\subsection{Inovação tecnológica como vantagem competitiva}

Inovação pode ser definida como ideias, sistemas, tecnologias, produtos, processos, serviços ou políticas que são novos e de relevante aplicação (SAWANG e UNSWORTH, 2011). Anteriormente, Besant e Tidd (2007) já afirmavam que o conceito de inovação se desdobra em inovação de produto (mudanças nas coisas - produtos ou serviços - oferecidas pelos agentes ao mercado) ou inovação de processo (mudanças nas formas como as coisas são criadas e colocadas no mercado), e as inovações podem fazer uma enorme diferença para organizações de todos os tipos e tamanhos, pois a lógica é simples: se elas não mudam o que oferecem ao mundo (produtos e serviços) ou como os criam e introduzem no mercado, elas arriscam ser superadas por concorrentes que o façam. Segundo os mesmos autores, os agentes econômicos atuantes no mercado em competição com outros e que sobrevivem só o fazem porque são capazes de mudanças regulares e focadas e, num aspecto mais amplo, as inovações são fortemente associadas com crescimento econômico dos países, constituindo uma parcela significativa desse crescimento desde o século XVIII. As inovações tecnológicas vêm tendo crescente valorização na dinâmica competitiva dos mercados e o desenvolvimento econômico e o progresso das nações tem sido cada vez mais associado ao domínio de conhecimentos que possibilitem produzir inovações tecnológicas, as quais constituem fator fundamental para a competitividade das empresas atuantes no mercado (GARNICA e TORKOMIAN, 2009). Inovações tecnológicas representam, as empresas, menos gastos e maior satisfação de clientes, gerando maiores receitas, e para a sociedade, progresso decorrente da introdução de novidade ou aperfeiçoamento no ambiente produtivo ou social, o que resulta em novos produtos, processos ou serviços (ARAÚJO et al, 2010).

Historicamente, essa forma de análise adquiriu maior relevância quando ocorreu a mudança de paradigma da sociedade industrial para a sociedade de conhecimento, em que este adquire maior importância na capacidade competitiva de países e empresas e a inovação tecnológica emerge como elemento de referência gerador de vantagem competitiva, pois, na sociedade de conhecimento, este é o principal fator estratégico de riqueza e poder para os países e organizações, passando a inovação tecnológica a ser um fator importante para a produtividade e o desenvolvimento econômico (SORIA et al, 2010).

A utilização intensiva de ciência, tecnologia e inovação numa economia, então, permite o aumento da capacidade competitiva, o que gera novos empreendimentos, empresas, empregos e marcas comerciais e, assim, a tecnologia é determinante na delimitação de vantagens comparativas dos países no comércio mundial, pois quanto mais inovação tecnológica um país produz, melhor sua posição econômica no comércio internacional. (MATIAS-PEREIRA, 2011).

Tal mudança de paradigma coloca o conhecimento e sua gestão como fatores relacionados à capacidade competitiva dos países e empresas no contexto atual, em que se observam fenômenos como globalização e aceleração do desenvolvimento tecnológico, destacando a inovação como elemento gerador de vantagem competitiva, passando o conhecimento científico a ser considerado um dos pilares de sustentação do desenvolvimento econômico (FERREIRA, SORIA e CLOSS, 2012).

Esse cenário, como já afirmavam anteriormente Fujino e Stal (2007), aponta para uma necessidade de crescente produção de conhecimentos científicos inovadores e, assim, as nações que buscam maior crescimento econômico precisam desenvolver capacidade de gerar conhecimento e processar informação, pois a 
inovação vem se tornando um dos principais fatores de competitividade das organizações.

Portanto, o desenvolvimento de inovação gera vantagem competitiva para os países e suas empresas, pois representa para eles uma resposta da ciência à sua busca cada vez maior por diferenciação, sendo chave para a sua sustentabilidade em um mercado altamente competitivo (CLOSS e FERREIRA, 2012) e, consequentemente, resulta em desenvolvimento econômico e verdadeiro progresso social para suas populações, que se beneficiam tanto da melhora de sua qualidade de vida pelo acesso às inovações, como da maior circulação de riquezas por elas proporcionada.

\subsection{Instituições de ensino superior e sua relação com empresas no Brasil}

O Conselho Nacional de Desenvolvimento Científico e Tecnológico (CNPq), agência do Ministério da Ciência, Tecnologia e Inovação (MCTI), divulga a cada dois anos um censo dos grupos de pesquisa vinculados a instituições de ensino superior de todo o país, por meio do Diretório Grupos de Pesquisa do CNPq (RAPINI e RIGHI, 2006).

Rapini e Righi (2006) publicaram estudo que tinha como objetivo apresentar informações do Diretório dos Grupos de Pesquisa do CNPq que podem ser exploradas na investigação da atuação das universidades em pesquisas científicas e sua interação com empresas no Brasil. A metodologia adotada foi uma análise dos dados do último censo divulgado pelo Diretório na época, de 2004, apresentando em termos gerais as informações disponíveis no diretório e algumas das possíveis agregações e formas de análise de tais dados, entre elas a natureza das instituições de ensino superior a que os grupos de pesquisa que compuseram o Censo, se públicas ou privadas. As autoras ressaltaram, na época, que embora o Diretório contemple uma pequena parcela da comunidade científica nacional, ele apresenta-se como instrumento auxiliar na investigação da atuação das universidades na produção de pesquisa científica. Como resultado, a pesquisa conclui que, na época, em termos institucionais, a preponderância foi de grupos de pesquisa vinculados a instituições de ensino superior públicas federais, que historicamente tendem a agregar um maior número de competências científicas.

Atualizando o estudo de Rapini e Righi (2006), tem-se que o último Censo do Diretório de Grupos de Pesquisa do CNPq se refere a dados do ano de 2014 (BRASILCNPq, 2014). Dele, é possível tirar algumas conclusões acerca da produção científica nacional em termos institucionais.

Em 2014 havia 35.424 grupos de pesquisa vinculados a 492 instituições de ensino superior, num total de 180.262 pesquisadores nesses grupos. 43,9\% desses grupos se concentram na região Sudeste, enquanto apenas $7,5 \%$ estão na região Centro Oeste. Entre as 127 instituições com pelo menos 200 doutores pesquisadores cadastrados no Censo de 2014, as instituições públicas de ensino superior da região sudeste são responsáveis, sozinhas, por 30,6\% de todos os grupos de pesquisa do país. Embora estejam relacionadas entre essas 127, a UFG - Universidade Federal de Goiás, a UNB - Universidade de Brasília, o IF Goiano - Instituto Federal Goiano e a UEG - Universidade Estadual de Goiás, todas instituições públicas, são responsáveis, juntas, por apenas 2,9\% dos grupos de pesquisa em atuação no Brasil (BRASIL-CNPq, 2014).

As instituições privadas também têm participação pequena no que diz respeito aos grupos de pesquisa. Analisando mais uma vez as 127 instituições com pelo menos 
200 doutores pesquisadores cadastrados no Censo de 2014 do Diretório de Grupos de Pesquisa do CNPq, entre pessoas jurídicas de direito privado, destacam-se empresas públicas de pesquisa que, embora privadas, têm capital formado por recursos públicos, como a EMBRAPA - Empresa Brasileira de Pesquisa Agropecuária e a EPAMIG - Empresa de Pesquisa Agropecuária de Minas Gerais, e as instituições privadas de ensino superior, as quais, juntas, são responsáveis por apenas $7 \%$ de todos os grupos de pesquisa do país. (BRASIL-CNPq, 2014).

Pela análise do censo do Diretório de Grupos de Pesquisa do CNPq pode-se concluir, portanto, que a atividade de pesquisa na área acadêmica, no Brasil, é de responsabilidade principalmente de instituições de ensino superior públicas, as quais praticamente metade se concentram na Região Sudeste.

\subsection{Relação universidade-empresa}

A gestão de conhecimento para gerar inovação é uma realidade mais próxima das grandes empresas (GOMES e ROCHA, 2011), o que, pelas características da maioria das empresas brasileiras, representa um problema. Segundo dados divulgados pelo Serviço Brasileiro de Apoio às Micro e Pequenas Empresas SEBRAE Nacional, do universo de cerca 5,4 milhões de empresas formais brasileiras, 99\% são pequenas (microempresas ou empresas de pequeno porte), as quais concentram suas atividades em comércio e serviços $(86,9 \%)$ e são responsáveis por $27 \%$ do Produto Interno Bruto - PIB do Brasil e 51,67\% dos empregos gerados (BRASIL-SEBRAE, 2015). O critério de classificação como pequena empresa é legal e leva em consideração o faturamento bruto anual, que está limitado a 3,6 milhões de reais, conforme art. 3ำ da Lei complementar 123/2006 (BRASIL, 2006). Essa característica explica o fato de suas atividades se concentrarem em comércio e serviços e não em indústria, porque esta última atividade demanda produção em escala, ou seja, fabricar em grande quantidade para obter preço de venda final baixo (SORIA et al, 2010) o que representa maior custo de produção, não suportado pelo faturamento baixo das pequenas empresas.

Outra pesquisa, divulgada pelo Instituto Brasileiro de Geografia e Estatística IBGE - de Estatísticas do Cadastro Central de Empresas - CEMPRE, divulgada em 2015 com dados referentes ao ano de 2013, informa que, desse universo de 5,4 milhões de empresas, apenas cerca de 640 mil ocupam mais de 10 pessoas (BRASILIBGE, 2015). E, ainda segundo o IBGE, na última Pesquisa de Inovação - PINTEC divulgada em 2013 com dados referentes a 2011, dessas que ocupam mais de 10 pessoas, apenas $35,7 \%$ implementarem produtos ou processos novos ou significativamente aprimorados (BRASIL-IBGE, 2013).

É interessante analisar os dados da PINTEC. Segundo o IBGE, ao justificar os aspectos da amostragem da pesquisa, a inovação no Brasil é um fenômeno raro e, por isso, os desenhos tradicionais de amostragem levariam a amostras que não representariam adequadamente a fração de empresas que implementaram inovações, o que levou à escolha de empresas que possuem maior probabilidade de serem inovadoras, baseado em vários critérios estatísticos. Por isso, aquelas que ocupam apenas até 10 pessoas nem foram consideradas na pesquisa (BRASIL-IBGE, 2013). Logo, da análise combinada dessas pesquisas conduzidas por entidades estatais brasileiras, se considerarmos o conjunto total de 5,4 milhões de empresas e que apenas cerca de 229 mil (35,7\% das que ocupam mais de 10 pessoas) implementaram inovações, temos como resultado que, de todas as empresas brasileiras, apenas 4,23\% implementam inovações de produto ou processo. 
Diante desse contexto econômico característico brasileiro de prevalência das pequenas empresas e em razão de seus limitados recursos financeiros, elas não têm condições de financiar atividades de pesquisa e desenvolvimento (P\&D) por conta própria e nem de suportar os riscos de tais atividades na busca de inovações ou de avaliar as opções tecnológicas para aumentar suas chances de acerto, e a aproximação com as universidades, que possuem a pesquisa como uma de suas atividades fim, apresenta-se como alternativa para antecipar oportunidades tecnológicas (BENEDETTI e TORKOMIAN, 2010).

Gomes e Rocha (2011) realizaram pesquisa em que foi constatado que as micro e pequenas empresas têm dificuldades em trabalhar estrategicamente, e tal problema não está relacionado à falta de informação, mas sim à falta de gestão estratégica das informações que se encontram disponíveis em abundância nas próprias empresas. Pelos resultados da pesquisa, constatou-se que as pequenas empresas possuem tendência competitiva proativa e procuram adotar inovação como prática de estratégia organizacional para vencer a concorrência, mas existem conflitos, como a não disponibilidade de uma estrutura adequada de apoio às atividades de monitoramento do ambiente externo e prospecção, por ausência de pessoal capacitado ao exercício de tais atividades, embora elas sejam facilitadas pelo contato direto do empresário com clientes e fornecedores, uma vez que as pequenas empresas possuem estrutura administrativa simples e flexível, a qual, se por um lado facilita bastante o fluxo de informação e comunicação interna, por outro pode comprometer a utilização de tais informações, que ficam em poder do principal dirigente, além da falta de recursos financeiros que respaldem as atividades de forma dinâmica e inovadora.

As empresas recorrem, então, à formação de alianças, parcerias ou redes para o desenvolvimento e inclusão de inovações no mercado, mais rápido e com menor custo, pois tais arranjos permitem combinação de competências, diluição do investimento em $P \& D$, dos custos e riscos, além de possibilitar às empresas terem acesso a benefícios que não obteriam sozinhas (ALBERTIN e AMARAL, 2010). Nesse contexto, destacam-se duas formas de organização desenvolvedoras e aplicadoras desses conhecimentos novos: a empresa, que visa o desenvolvimento de novos produtos e as instituições que atuam em pesquisa e desenvolvimento (P\&D), mas não implementam primordialmente as inovações tecnológicas como produtos: universidades e institutos de pesquisa. (GARNICA e TORKOMIAN, 2009).

Isso se dá porque, nos últimos anos, a ideia de inovação migrou de um significado mais estreito de criação de novos produtos e processos para uma acepção mais ampla de inovação organizacional e de mercado. A análise do processo inovador também mudou, deixando de se concentrar apenas na geração linear de novos saberes e passando a abordar o desenvolvimento de novas formas de produzir, aplicar e distribuir o conhecimento, com ênfase para as parcerias tecnológicas firmadas entre empresas e universidades. (COSTA; PORTO; FELDHAUS, 2010). A transferência de tecnologia das universidades para o setor produtivo é um processo complexo que possibilita o emprego de diversas estratégias e está sujeito às características inerentes de cada setor da economia, e é crescente o número de contratos celebrados entre empresas e universidades como resultado de um maior interesse das primeiras em desenvolver novos meios de gestão em P\&D (BENEDETTI e TORKOMIAN, 2010).

As instituições públicas de ensino superior, no campo da pesquisa e da inovação tecnológica, por exemplo e como se verá mais detalhadamente adiante, podem atuar em parceria com empresas privadas voltadas à pesquisa científica e 
voltadas ao desenvolvimento de novas tecnologias, com potencial de impactar a sociedade como um todo, na modalidade de parcerias público-privadas e, nesse tipo de ambiente, o principal objetivo deixa de ser o lucro financeiro, característica das empresas privadas e em algumas empresas estatais, para algo menos palpável, mas relevante: a criação e circulação de conhecimento, circunstância que não pode ser esquecida quando se analisam parcerias entre o Estado e as entidades de pesquisa (ALMEIDA, JURKSAITIS e MOTA, 2015).

Tais características do sistema empresarial brasileiro enquadram-se perfeitamente naquilo que Etzkowitz e Leydesdorff (2000) chamaram de ambiente de inovação, ou innovative environment, no já clássico estudo em que propuseram o chamado modelo da Hélice Tripla, ou Triple Helix, o qual influenciou vários estudos brasileiros sobre a relação universidade-empresa e que se passa a detalhar.

\subsection{O Modelo da Hélice Tripla}

O modelo teórico da Hélice Tripla, proposto por Etzkowitz e Leydesdorff (2000), se baseia na percepção de que a universidade desempenha um papel relevante em produção de inovação em uma sociedade de conhecimento e, a partir disso, diferencia-se analiticamente em relação a modelos teóricos anteriores que ou atribuíam às empresas a liderança no desenvolvimento de inovações (sistemas nacionais de inovação nos Estados Unidos, na época), ou privilegiavam o papel do Estado nessa atividade (modelo do Triângulo de Sábato).

Etzkowitz e Leydesdorff propõem, então, um novo modelo baseado numa rede de comunicações e expectativas que remodela os arranjos institucionais entre universidades, empresas e governo. Justificam ao observar que enquanto as forças armadas diminuem sua participação no processo inovativo, a universidade surge em evidência nas estruturas institucionais das sociedades contemporâneas e, consequentemente, as redes de relacionamento entre universidades, empresas e governo se transformaram, substituindo o modelo trilateral de "Power Elite" da Guerra Fria por uma sobreposição de comunicações reflexivas que crescentemente remodelam a infraestrutura, o que, não surpreendentemente, provocou um debate internacional sobre o papel apropriado das universidades no processo de transferência de tecnologia e conhecimento.

Então, diante dessa nova perspectiva, Etzkowitz e Leydesdorff afirmam que a evolução dos sistemas de inovação, e a dúvida a respeito de qual o melhor caminho a ser tomado nas relações universidade-empresa, refletem na variação de arranjos institucionais de universidades, empresas e governo. Numa primeira versão, por eles chamada de "Triple Helix I", o Estado se sobrepõe em relação às universidades e empresas, dirigindo as relações entre elas, citando como exemplos desse modelo de arranjo institucional o que era praticado na antiga União Soviética e no Leste Europeu durante o regime socialista. Os autores afirmam que esse modelo é visto, de maneira geral, como um modelo de desenvolvimento falido, uma vez que proporciona muito poucas iniciativas "de baixo para cima", o que acaba por desencorajar a prática de inovação, ao invés de encorajá-la. Numa segunda versão ("Triple Helix II"), as três esferas (universidades, empresas e Estado) são claramente distintas e separadas por fortes limites, mas existe a livre interação entre elas, como se observava, na época (2000), na Suécia e nos Estados Unidos. Nesse modelo, porém, vigora uma política "laissez-faire", que é defendida como forma de diminuir a participação do Estado exposto no modelo "Triple Helix I". Finalmente, a última versão do modelo, "Triple Helix III", gera uma infraestrutura de conhecimento em termos de esferas institucionais que 
se sobrepõem, com cada uma assumindo o papel da outra e com organizações híbridas emergentes nas interfaces. Os autores afirmam que muitos países têm buscado aplicar esse último modelo de algum modo, com o objetivo de criar o que eles chamam de ambiente de inovação, caracterizado por criação de empresas spinoff nas universidades, iniciativas trilaterais de desenvolvimento econômico baseado em produção de conhecimento, alianças estratégicas entre empresas de diferentes portes econômicos e que atuam em diferentes áreas com diferentes níveis de tecnologia, laboratórios do governo e grupos de pesquisa acadêmicos. Segundo Etzkowitz e Leydesdorff (2000), tais arranjos organizacionais têm sido encorajados, mas não controlados, pelos governos, que estabelecem novas "regras de jogo" por meio de assistência financeira direta ou indireta e por leis que incentivam a formação desses arranjos.

Para os autores, a Tripla Hélice consiste, então, em um modelo analítico para descrição da variedade de arranjos e políticas institucionais e explicação de suas dinâmicas de funcionamento, buscando identificar quais são as unidades de operação que interagem quando um sistema de inovação é formado e como tal sistema pode ser especificado. Na opinião dos autores, ocorrências como "sistemas nacionais de inovação", "sistemas de pesquisa em transição", ou "sistema de pesquisa pósmoderno", são indicativos de um fluxo, reorganização e reforço do papel do conhecimento e da inovação na economia e na sociedade.

Ao tratar das implicações do modelo da Hélice Tripla, Etzkowitz e Leydesdorff (2000) afirmam que o modelo repercute não apenas no relacionamento entre universidades, empresas e governo, mas também internamente em cada uma dessas esferas. A universidade se transforma de simples instituição de ensino em uma que combina esta função com a de pesquisa. Com relação às demais, o modelo resulta que os arranjos entre governo e empresas não se restringem mais a governos nacionais e setores industriais específicos. Os governos podem atuar em níveis nacional, regional e até internacional. As empresas adotam posturas globais tanto dentro de estruturas formais quanto em alianças. Blocos comerciais como a União Europeia, o NAFTA e o MERCOSUL proporcionam novas opções de quebra de bloqueios comerciais sem sacrificar as vantagens competitivas.

O modelo teórico da Hélice Tripla apresentado por Etzkowitz e Leydesdorff (2000) tem sido referenciado em muitas pesquisas acadêmicas produzidas no Brasil e que tratam da relação entre universidades, empresas e governo no ambiente de inovação, como mencionado por Closs e Ferreira (2012) e por Lemos, Cário e Melo (2015). Anteriormente, Dagnino (2003) já tratava de tal ambiente de inovação ao mencionar elementos que reforçam a importância das relações entre os atores que nele atuam, citando diversos outros pesquisadores que, embora mantendo uma ênfase na empresa, evidenciam a capacidade que esta tem de ter de estabelecer relações virtuosas com os demais elementos que atuam no ambiente de produção de inovação. $E$, entre estes, no modelo da Hélice Tripla, a universidade assume a posição de elemento privilegiado do ambiente inovativo e, embora não seja situada no centro do Sistema Nacional de Inovação, lugar ocupado pela empresa, a universidade é considerada fator indispensável de competitividade sistêmica.

Dagnino (2003) destacava, ainda, a formação do Sistema Nacional de Inovação nesses parâmetros, salientando o contexto em que se dá a formação dessa política da relação universidade empresa, em que a desregulamentação da economia e crescente exposição das empresas nacionais à concorrência externa teria como resultado esperado um impacto positivo na competitividade dessas mesmas empresas, o que provocou uma percepção nas universidades, que já sofriam 
progressivos cortes orçamentários, que a mudança em curso demandava, também, uma mudança significativa de seu próprio comportamento. As comunidades de pesquisa e os empresários, então, passaram a sugerir medidas de política de inovação que levassem a um aumento da competitividade das empresas locais, com o objetivo de criar uma nova dinâmica a partir de empresas de base tecnológica, o que viria impactar os outros atores envolvidos no processo, especialmente as universidades, a quem as empresas submetidas à concorrência internacional recorreriam em busca de conhecimento.

$\mathrm{Na}$ esteira desse pensamento, a Lei de Inovação (Lei 10.973/2004) foi editada com os objetivos de constituir ambiente propício para parcerias estratégicas entre universidades, institutos tecnológicos e empresas, estimular a participação das Instituições de Ciência e Tecnologia - ICT - no processo de inovação, conceder incentivos ao pesquisador-inventor vinculado à ICT e apropriar conhecimentos gerados nas ICT em favor das empresas (ARAÚJO et al, 2010). Fujino e Stal (2007) indicam que a edição da Lei de Inovação acompanha um movimento internacional em que se enfatiza a necessidade de um trabalho dinâmico entre os agentes da Hélice Tripla proposta por Etzkowitz e Leydesdorff, de forma a viabilizar o desenvolvimento tecnológico local, regional ou nacional e, assim, políticas estatais de incentivo à relação universidade-empresa resultaram em práticas mais ou menos homogêneas e bem-sucedidas em Israel, nos Estados Unidos, em Taiwan e no Brasil.

Soria et al (2010), por sua vez, salientam que, nessa conjuntura, tem-se testemunhado mudanças no ambiente regulatório da transferência de tecnologia universidade-empresa, na definição de metas de pesquisa acadêmica (aumento de exigência de publicações e de contribuições com o desenvolvimento econômico) e suporte público à ciência. Com isso, procura-se criar esse ambiente de inovação em que a universidade desempenha papel fundamental na formação de profissionais qualificados, no acompanhamento do estado da arte em nível internacional, na realização de pesquisas que resultem em processos e produtos inovadores, na manutenção de um ambiente interno estimulante para o empreendedor e na transferência de resultados de pesquisa ao setor produtivo.

Ipiranga, Freitas e Paiva (2010) afirmam que a inovação é um processo cumulativo e interativo e a cooperação dos atores da Hélice Tripla em termos de Sistemas de Inovação resulta em um arranjo organizacional mais evoluído, o que teria sido o evento chave para a criação de uma "universidade empreendedora" e de condições em prol de uma sociedade baseada em conhecimento, integrando o desenvolvimento econômico e social local e regional. Vieira da Silva (2010) acrescenta que, sob a égide da Hélice Tripla, têm surgido no Brasil novos arranjos sociais para buscar uma mudança no atual cenário econômico, entre eles núcleos de inovação tecnológica - NIT, polos tecnológicos, centros de modernização empresarial e incubadoras de empresas nas universidades, as quais constituem instituições híbridas que internalizam o relacionamento entre as três esferas da Hélice, estimulando e criando um espaço de interação.

Voltando ao estudo de Closs e Ferreira (2012), tem-se a afirmação de que no modelo da Hélice Tripla o ambiente de inovação proveniente da inter-relação entre os atores da Hélice resulta também em uma ampliação do papel da universidade, que passa a ser fonte de geração e difusão de conhecimento e inovações, agregando-se a necessidade de alinhamento da produção acadêmica com as demandas da sociedade, num cenário de dependência mútua com as empresas, detentoras da lógica de uso de inovações com finalidade comercial, mas que buscam nas 
universidades o conhecimento necessário para a produção de tais inovações, uma resposta da ciência à sua busca por maior diferenciação no mercado competitivo.

Já no entendimento de Eberhart e Pascuci (2014), no modelo da Hélice Tripla a universidade produz o conhecimento, a empresa cria o produto e o estado contribui com recursos e incentivos fiscais para a realização de tais atividades e, em tal perspectiva, a universidade seria o ator mais representativo na Hélice, na medida em que exerce papel de interseção entre os demais atores. Contudo, em organizações universitárias, o processo decisório multifacetado, burocrático e centralizador, o que influencia as decisões sobre o que e como fazer, e a ambiguidade das preferências e objetivos somada à natureza qualitativa e subjetiva da área acadêmica e aos fatores políticos que vão influenciar as decisões, afetam esse movimento de interseção e o cumprimento de seu papel na Hélice Tripla (EBERHART e PASCUCI, 2014).

\section{CONSIDERAÇÕES FINAIS}

A partir das referências utilizadas no presente trabalho, tem-se que o fato de a maioria das empresas brasileiras serem de pequeno porte econômico dificulta que estas realizem investimentos em pesquisas de produção de inovações tecnológicas, em razão da falta de recursos, o que dificulta a competição com as grandes empresas, que possuem melhores condições econômicas para investir em pesquisas científicas.

Nesse cenário, no Brasil a produção de tecnologia de fronteira acaba por ficar a cargo das pesquisas realizadas por universidades, principalmente as de natureza pública, que concentram a maior parte da produção científica do país.

O modelo teórico da Hélice Tripla, que propõe a criação de um ambiente favorável para aproximação das universidades e das empresas para transferência de tecnologia por intermédio de incentivos concedidos pelo Estado, apresenta-se como boa solução para proporcionar às empresas de pequeno porte econômico terem acesso a inovações tecnológicas que lhes forneçam vantagens competitivas no mercado. 


\section{Technological innovation, university-industry relationship and Triple Helix model}

\section{Abstract}

This paper intends accomplish a little cut of scientific literature about technological innovation as a competitive advantage, the role of universities in such an environment and their relationship to industries for technology transfer in the context of the Triple Helix model, proposed by Etzkowitz \& Leydesdorff (2000). Therefore, papers dealing with the issues presented and recently published were used as references. As a result, in Brazil the industries have characteristics that hinder investment in scientific research, which eventually concentrate on research conducted by universities much of the production of technological innovations. The Triple Helix model, dealing with the relationship between universities, industries and government, It aims to create favorable environment for closer relations between universities and industry and facilitate the transfer of technology from academia to the private initiative.

Key words: innovation; university-industry relationship; Triple helix

\section{REFERÊNCIAS}

BRASIL. 2006. Lei complementar 123, de 14 de dezembro de 2006. Diário Oficial [da] República Federativa do Brasil, Poder Executivo, Brasília, DF, 15 dez. 2006. Disponível em: <http://www.planalto.gov.br/ccivil_03/leis/LCP/Lcp123.htm>. Acesso em: 29 abr. 2016.

. Conselho Nacional de Desenvolvimento Científico e Tecnológico - CNPq.

Censo do Diretório dos Grupos de Pesquisa no Brasil. Brasília, DF, 2014. . Instituto Brasileiro de Geografia e Estatística - IBGE. Estatísticas do Cadastro Central de Empresas. Rio de Janeiro, RJ, 2015. . Instituto Brasileiro de Geografia e Estatística - IBGE. Pesquisa de Inovação. Rio de Janeiro, RJ, 2013.

Serviço Brasileiro de Apoio às Micro e Pequenas Empresas SEBRAE. Participação das Micro e Pequenas Empresas na Economia Brasileira - Relatório Executivo. Brasília, DF, 2015.

ALBERTIN, Eduardo Vicente; AMARAL, Daniel Capaldo. Contexto da parceria como qualificador da gestão de projetos universidade-empresa. Produção, v. 20, n. 2, p. 224-236, abr./jun. 2010. 
ALMEIDA, Fernando Dias Menezes de Almeida; JURKSAITIS, Guilherme Jardim; MOTA, Carolina. Parcerias empresariais do Estado para a inovação tecnológica. In: JUSTEN FILHO, M.; SCHWIND, R. (Coord.). Parcerias público-privadas Reflexões sobre os 10 anos da Lei 11.079/2004. São Paulo: Revista dos Tribunais, 2015, p. 609-625.

ARAÚJO, Elza Fernandes; BARBOSA, Cynthia Mendonça; QUEIROGA, Elaine dos Santos; ALVES, Flávia Ferreira. Propriedade Intelectual: proteção e gestão estratégica do conhecimento. R. Bras. Zootec., v.39 (supl. especial), p.1-10, 2010.

BENEDETTI, Mauricio Henrique; TORKOMIAN, Ana Lúcia Vitale. Uma análise da influência da cooperação universidade-empresa sobre a inovação tecnológica. Gest. Prod., São Carlos, v. 17, n. 4, p. 145-158, 2010.

BESSANT, John; TIDD, Joe. Innovation and Entrepreneurship. Londres: John Wiley \& Sons, Ltd., 2007.

CLOSS, Lisiane; FERREIRA, Gabriela Cardozo. A transferência de tecnologia universidade-empresa no contexto brasileiro: uma revisão de estudos científicos publicados entre os anos 2005 e 2009. Gest. Prod., São Carlos, v. 19, n. 2, p. 419432, 2012.

CLOSS, Lisiane; FERREIRA, Gabriela Cardozo. A transferência de tecnologia universidade-empresa no contexto brasileiro: uma revisão de estudos científicos publicados entre os anos 2005 e 2009. Gest. Prod., São Carlos, v. 19, n. 2, p. 419432, 2012.

COSTA, Priscila Rezende; PORTO, Geciane Silveira; FELDHAUS, Diogenes. Gestão da Cooperação Empresa-Universidade: o Caso de uma Multinacional Brasileira. RAC - Revista de Administração Contemporânea, Curitiba, v.14, n.1, art.6, p. 100-121, jan./fev. 2010.

DAGNINO, Renato. A relação universidade-empresa no Brasil e o "argumento da Hélice Tripla”. Revista Brasileira de Inovação, Campinas, v. 2, n. 2, p. 267-307, jul./dez. 2003.

EBERHART, Maria Elizete; PASCUCI, Lucilaine. O processo decisório e suas implicações na cooperação universidade, empresa e governo: um estudo de caso. Revista GUAL, Florianópolis, v. 7, n. 2, p. 221-242, mai. 2014.

ETZKOWITZ, Henry; LEYDESDORFF, Loet. The dynamics of innovation: from National Systems and "Mode 2" to a Triple Helix of university-industry-government relations. Research Policy, v. 29, n. 2, p. 109-123, fev. 2000.

FERREIRA, Gabriela Cardozo; SORIA, Alessandra Freitas; CLOSS, Lisiane. Gestão da interação universidade-empresa: o caso PUCRS. Revista Sociedade e Estado, v. 27 , n. 1, p. 79-94, jan./abr. 2012. 
FUJINO, Asa; STAL, Eva. Gestão da propriedade intelectual na universidade pública brasileira: diretrizes para licenciamento e comercialização. Revista de Negócios, Blumenau, v. 12, n. 1, p. 104 - 120, jan./mar. 2007.

GARNICA, L.; TORKOMIAN, A. Gestão de tecnologia em universidades: uma análise do patenteamento e dos fatores de dificuldade e de apoio à transferência de tecnologia no Estado de São Paulo. Gest. Prod., São Carlos, v. 16, n. 4, p. 624-638, out./dez. 2009.

GOMES, Suely; ROCHA, Jaqueline. Gestão da informação: o caso das empresas participantes do programa de incubação de empresas da Universidade Federal de Goiás. Palabra Clave, La Plata, v. 1, n. 1, p. 21-39, out. 2011.

IPIRANGA, Ana Sílvia Rocha; FREITAS, Ana Augusta Ferreira de; PAIVA, Thiago Alves. O empreendedorismo acadêmico no contexto da interação universidade empresa - governo. Cadernos EBAPE. Rio de Janeiro, v. 8, n. 4, p. 676-693, dez. 2010.

LEMOS, Dannyela da Cunha; CÁRIO, Silvio Antônio Ferraz; MELO, Pedro Antônio de. Processo de interação universidade-empresa em Santa Catarina para o desenvolvimento inovativo: o caso da UFSC, FURB, UDESC e UNIVALI. Revista de Ciências da Administração, v. 17, n. 43, p. 37-54, dez. 2015

MATIAS-PEREIRA, José. A gestão do sistema de proteção à propriedade intelectual no Brasil é consistente? RAP - Revista de Administração Pública. Porto Alegre, v. 45, n. 3, p. 567-590, mai./jun. 2011.

RAPINI, Márcia Siqueira; RIGHI, Hérica Morais. O Diretório dos Grupos de Pesquisa do CNPq e a Interação Universidade-Empresa no Brasil em 2004. Revista Brasileira de Inovação, Campinas, v. 5, n. 1, p. 131-156, jan./jun. 2006.

SAWANG, Sukanlaya; UNSWORTH, Kerrie L. A model of organizational innovation implementation effectiveness in small to medium firms. International Journal of Innovation Management, v. 15, n. 5, p. 989-1011, 2011.

SORIA, Alessandra Freitas; FERREIRA, Gabriela Cardozo; PERIN, Marcelo Gattermann; SAMPAIO, Cláudio Hoffmann; ALEMÁN, José Luis Munuera. Geração de patentes em universidades: um estudo exploratório. R. Adm. FACES Journal Belo Horizonte, v. 9, n. 3, p. 95-116, jul./set. 2010. 
